\title{
TCO WORK FUNCTION TUNING BY NANODIAMONDS
}

\author{
1'Jan ČERMÁK, 'Daria MILIAIEVA, ${ }^{2}$ Aurelien SOKENG DJOUMESSI, ${ }^{2}$ Harald HOPPE, \\ 'Štěpán STEHLÍK
}

${ }^{1}$ Instutite of Physics, Czech Academy of Sciences, Prague, Czech Republic, EU, cermaki@fzu.cz

${ }^{2}$ Center for Energy and Environmental Chemistry Jena, Friedrich-Shiller-University Jena, Germany, EU, harald.hoppe@uni-jena.com

https://doi.org/10.37904/nanocon.2020.3688

\begin{abstract}
High-pressure high-temperature nanodiamonds (NDs) with hydrogen and oxygen surface termination are deposited on ITO coated glass substrates as well as on gold layers. Scanning probe microscopy is used for characterization of their microscopic electrical and electronic properties. Namely, conductive AFM was used to detect electric conductivity of NDs which prove that they act similarly to bulk diamond: hydrogen terminated NDs are electrically conductive whereas oxygen terminated NDs are electrically insulating. Work function (WF) of the ND-TCO systems were characterized by Kelvin probe force microscopy. It turned out, that WF of NDs on ITO is not stable in time. WF of H-NDs on ITO is changed by $150 \mathrm{mV}$ to lower WF within 17 days. WF of O-NDs is varying in the range $-250 \mathrm{mV}$ up to $+650 \mathrm{mV}$ without any monotonous trend, which reflects their insulating character.
\end{abstract}

Keywords: Nanodiamonds, ITO, surface termination, Kelvin probe force microscopy, conductive atomic force microscopy, work function

\section{INTRODUCTION}

Photovoltaics covers large family of specific designs and material composition. Apart from the mostly used silicon photovoltaic (PV) cells, nowadays there is a remarkable increase in development of organic and perovskite PV cells. Their basic design consists of an opaque metal back electrode, active medium, and a transparent top electrode. To achieve best PV operation, the energy levels of the electrodes must be optimized for the best charge carrier collection [1]. The most common material for the transparent electrode is indiumtin-oxide (ITO). To enhance the PV operation additional interfacial layers are deposited between the active material and the ITO electrode like PEDOT:PSS [2] that is a hole conducting material and acts as an electron blocking layer. However, PEDOT:PSS is not an ideal solution as it is hygroscopic and acidic which causes degradation of the active medium [3]. Therefore, there is a demand to find a replacement [4].

Diamond is a well-known material due to its high durability, chemical stability, high thermal conductivity, or optical transparency across the visible spectrum due to its large energy bandgap (5.5 eV) [5]. From this point of view, diamond is a wide bandgap semiconductor that is electrically insulating at ambient conditions in its intrinsic form. However, it may become electrically conductive when doped or when its surface is terminated by hydrogen atoms [6]. In the latter case a thin $(\sim 10 \mathrm{~nm})$ hole-conducting layer is formed on the surface. Yet, the conductivity of such a layer is not high enough (resistivity in the order of $10^{4} \mathrm{kOhm} / \mathrm{sq}$ [7]) to be used as an effective electrode in PV cell directly. 
Preliminary experiments indicated that nanodiamonds (NDs) inherit all these extraordinary properties of diamond including the hole conductivity when hydrogen terminated [8]. Moreover, computational modelling indicates that in a system of a diamond nanocrystal and an organic dye, there occurs a space separation of the highest occupied molecular orbital (HOMO) and the lowest unoccupied molecular orbital (LUMO) [9]. Thus, NDs are of high interest for many applications including photovoltaics for the use both as a hole conducting interlayer and as part of the active medium. They are available in large quantities at low pricing, their surface termination can be modified similarly to the bulk diamond and their processing is not technically demanding as they are purchased in the form of a powder or a colloid solution.

In this work the electronic properties of ND layers on ITO are characterized. Conductive atomic force microscopy (C-AFM) is used for testing their electrical conductivity and Kelvin probe force microscopy (KPFM) studies the change in surface potential that usually corresponds to a change in WF. The study is focused on NDs with two surface termination: hydrogenated NDs (H-NDs) and oxidized NDs (O-NDs).

\section{EXPERIMENTAL DETAILS}

High-pressure high-temperature NDs with a median size of $18 \mathrm{~nm}$ and size range $0-25 \mathrm{~nm}$ were purchased from Microdiamant AG. Initially, they were annealed in air at $450{ }^{\circ} \mathrm{C}$ for 5 hours. Hydrogenation was done in hydrogen atmosphere at atmospheric pressure at $800{ }^{\circ} \mathrm{C}$ for 6 hours. The colloid solutions were prepared by dissolving $1 \mathrm{mg}$ of NDs in $1 \mathrm{ml}$ of deionized water, ultrasonicating for 1 hour (at $\sim 50 \mathrm{~W}$ ) and decanting for 12 hours. The supernatant upon the decantation was used for samples preparation.

C-AFM was performed on an Icon system by Bruker equipped by a PeakForce TUNA (PF-TUNA) module using Multi75E-G probes (BudgetSensors). The system was operating in the PeakForce TUNA mode and for current detection the Contact current channel was used. The PeakForce setpoint was set to $18 \mathrm{nN}$ and the bias voltage applied to the sample was $-2.5 \mathrm{~V}$. For PF-TUNA experiment NDs were drop-cast on a fresh thermally evaporated gold film on a glass substrate.

KPFM experiments were performed by an NTEGRA Prima system (NT-MDT) equipped with an infrared laser in the detection system using Multi75E-G probes (BudgetSensors). Here, ITO covered glass substrates were cleaned by ultrasonication in acetone, isopropanol and deionized water and dried by nitrogen flow. NDs were drop-cast on the surface and let evaporate on a hot plate $\left(60^{\circ} \mathrm{C}, 1 \mathrm{~min}\right)$. As the measurements were repeated within several weeks and consequently several probes (though of the same type) with different properties were used, we compare here the difference of surface potential between the NDs and the value measured on bare ITO surface on the same sample within the same measurement session, thus with the same tip and microscope parameters.

\section{RESULTS AND DISCUSSION}

Because NDs are not firmly attached to the ITO surface, the common conductive AFM method in contact mode cannot be used. The PF-TUNA regime is more suitable as the contact force is controlled at each probe approach-retract cycle [8]. Topography images (Figure 1) show that the measurement is performed on dense ND layers of O-NDs (Figure 1a) and H-NDs (Figure 1b) without protrusion to the underlying substrate. Topography of both samples are quite similar, the individual NDs are distinguishable by the AFM tip. In contrast, corresponding local current maps present a huge difference in electric conductivity. In similarity to bulk diamond, O-NDs are electrically insulating (Figure 1c; white color corresponds to electric current close to zero), while H-NDs are electrically conductive (Figure 1d; dark color corresponds to electric current below the bottom limit of the z-scale). Moreover, the electric conductivity is homogeneously detected on all the H-NDs, which is different from the data in literature, where only few of H-NDs were conductive [8]. This indicates that the used method of thermal hydrogen termination is more efficient than the plasma method used in [8]. 


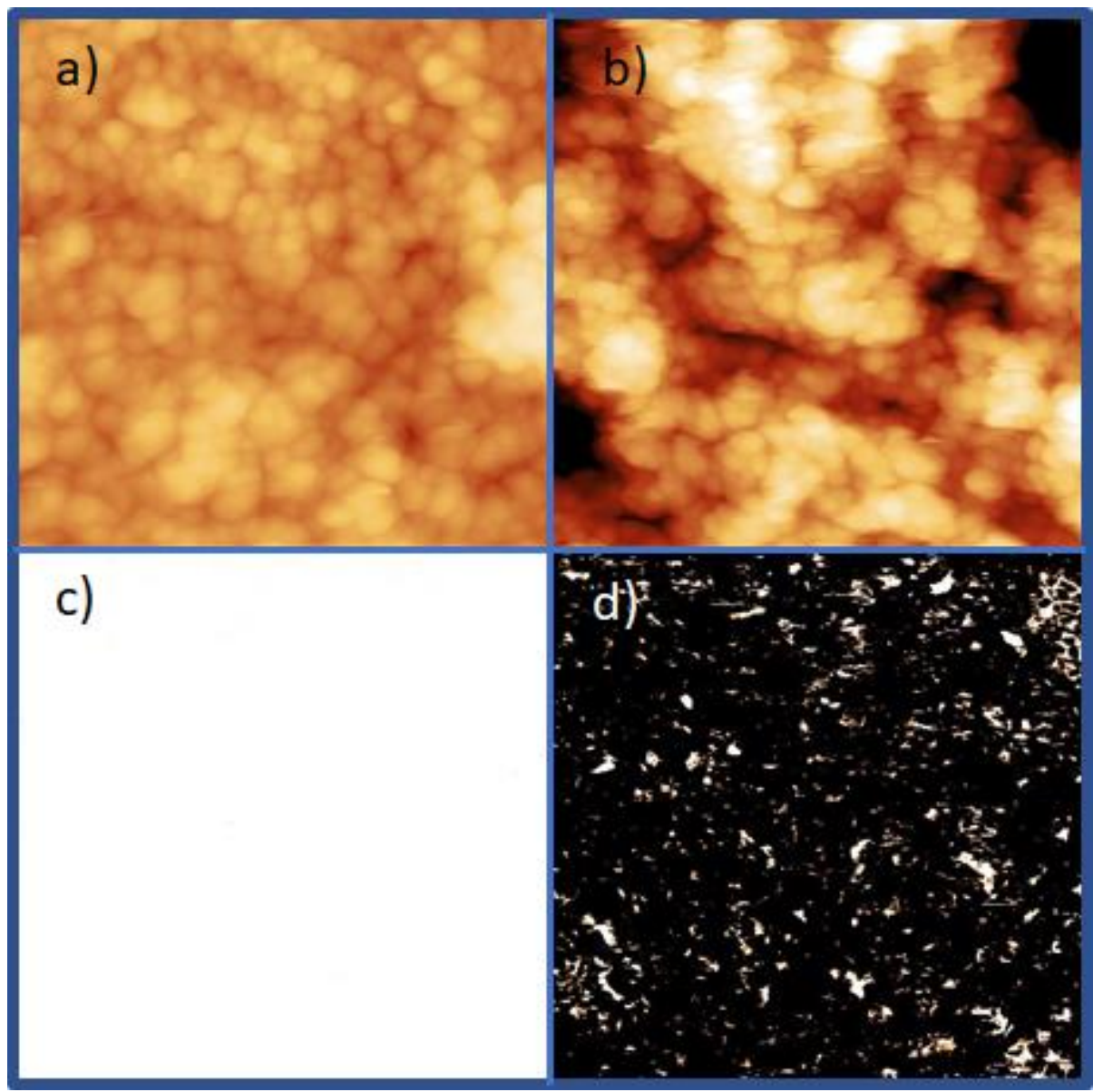

Figure 1 AFM topography (a, b) and local current maps (c, d) of O-NDs $(a, c)$ and H-NDs (b, d). Z-scale for topography images is $150 \mathrm{~nm}, \mathrm{z}$-range for local current maps are from $-5 \mathrm{pA}$ to $0 \mathrm{pA}$.

For PV applications it is important to know and tune the work function of the PV systems and components. The WF of ITO (and hence the measured surface potential by KPFM) is known to vary within a range 4.1 to $4.7 \mathrm{eV}$ when measured under vacuum by UPS or XPS [10]. Under ambient conditions, like in the case of KPFM or Kelvin Probe the obtained WF is typically higher: up to $5.2 \mathrm{eV}$ [11]. It is also known that its WF may be influenced by as much as $0.8 \mathrm{eV}$ by surface treatments [10]. Our control KPFM measurement showed that the ITO WF value is very close to the one of a gold reference, that is known to be $5.0 \mathrm{eV}$ [12]. However, the measured value was different at each measurement session and varied also from sample to sample (Figure 2a). Therefore, in this work we compare and watch a trend in time of the difference between the WF of NDs and the WF if bare ITO on the same sample. The results are presented in Figure $\mathbf{2 b}$. Note, that in our experimental setup the positive value of the difference corresponds to a decrease in WF and vice versa.

Right after the deposition the WF of H-NDs is approx. $50 \mathrm{mV}$ lower than the one of ITO. During the period of 17 days the WF of H-NDs exhibit continuous differentiation from the WF of ITO up to approx. $150 \mathrm{mV}$ that corresponds to an effective WF of $4.85 \mathrm{eV}$. This is close to a value $4.9 \mathrm{eV}$ reported for bulk hydrogenated diamond surface [13].

O-NDs follow a trend that is expectable for an electrically insulating material: WF is not properly defined and thus the KPFM results display somehow random value at which the measurement feedback control is stabilized, possibly influenced by a trapped electrostatic charge. Both effects are not under control and, therefore, each additional measurement provides non-predictable results. 

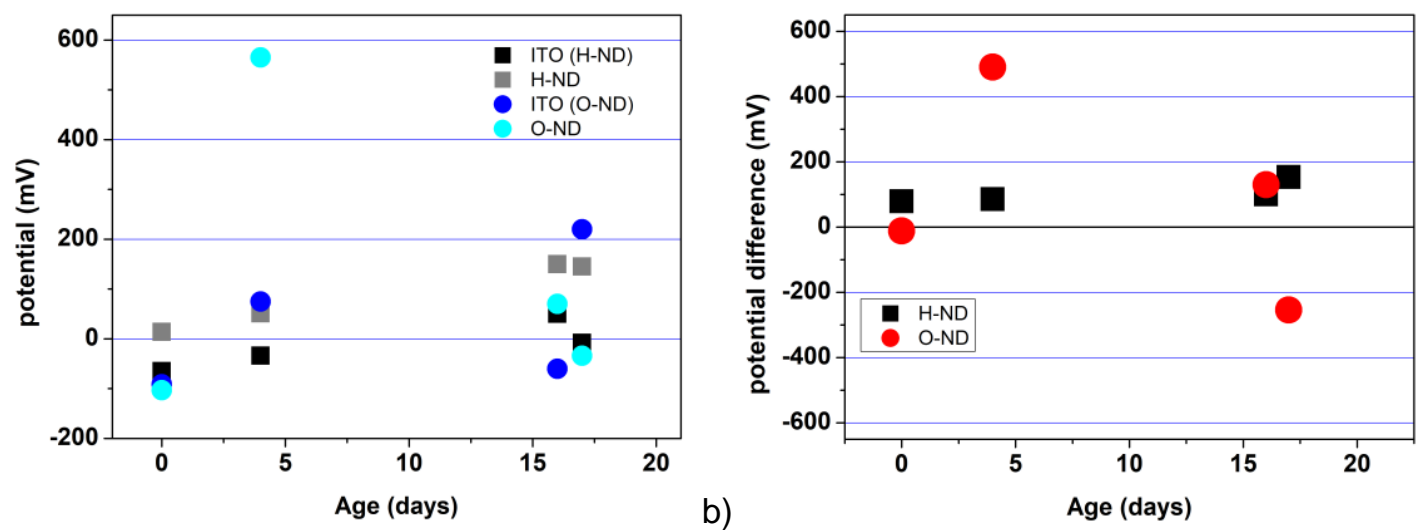

Figure 2 a) Potential values as measured by KPFM using a PtIr coated silicon tip on NDs and on the bare ITO on the corresponding substrates. b) Potential difference between the measured surface potential on ITO and on NDs as a function of time and ND surface termination.

From the data above it seems that $\mathrm{H}-\mathrm{NDs}$ are the more promising candidate for application in $\mathrm{PV}$ as a hole transporting layer as they maintain electric conductivity and the WF of the ITO/H-NDs system is more stable in time. Also the results on O-NDs are important for further research, as recently, it was demonstrated that not specifically H-NDs or O-NDs, but their mixed surface termination provides the best opto-electronic response when interacting with polypyrrole [14]. Further experiments to study the details of interaction of NDs with ITO and other materials relevant for PV are in preparation.

\section{CONCLUSION}

We performed microscopic electronic characterization of H-NDs and O-NDs by means of PF-TUNA and KPFM on Au and ITO substrates, respectively. By PF-TUNA we showed that similarly to bulk diamond, properly hydrogenated H-NDs kept the surface conductivity down to nanoscale while the oxidized O-NDs remained insulating. These properties were further reflected in the KPFM characterization. It was demonstrated that deposition of differently surface terminated NDs could effectively modify the WF of the system. H-NDs shifted WF close to a common WF value reported for hydrogen terminated diamond, thus acting as a conductive capping layer. In contrast, the deposition of O-NDs resulted in scattered WF values most probably due to their insulating properties and subsequent poor definition of their WF with possible contribution of an uncontrolled electrostatic charging effect.

\section{ACKNOWLEDGEMENTS}

This study was supported by the Czech Science Foundation (GACR) grant number 20-20991J and by the Ministry of Education, Youth and Sports of the Czech Republic (MEYS) under the project

CZ.02.1.01/0.0/0.0/16_019/0000760 (SOLID21). CzechNanoLab project LM2018110 funded by MEYS CR is gratefully acknowledged for the financial support of the measurements/sample fabrication at LNSM Research Infrastructure.

\section{REFERENCES}

[1] OPITZ, A., FRISCH, J., SCHLESINGER, R., WILKE, A. and KOCH, N. Energy level alignment at interfaces in organic photovoltaic devices. Journal of Electron Spectroscopy and Related Phenomena. [online] Oct. 2013, vol. 190, pp. 12-24. Available from: https://doi.org/10.1016/j.elspec.2012.11.008.

[2] KO, C.J., LIN, Y.K., CHEN, F.C. and CHU, C.W. Modified buffer layers for polymer photovoltaic devices. Applied Physics Letters. [online]. Feb. 2007, vol. 90, no. 6, p. 063509. Available from: https://doi.org/10.1063/1.2437703. 
[3] LIN, Y. et al. 17\% Efficient Organic Solar Cells Based on Liquid Exfoliated WS 2 as a Replacement for PEDOT:PSS. Advanced Materials. [online]. Nov. 2019, vol. 31, no. 46, p. 1902965. Available from: https://doi.org/10.1002/adma.201902965.

[4] NEOPHYTOU, M. et al. High mobility, hole transport materials for highly efficient PEDOT:PSS replacement in inverted perovskite solar cells. Journal of Materials Chemistry C. [online]. 2017, vol. 5, no. 20, pp. 4940-4945. Available from: https://doi.org/10.1039/C7TC00858A.

[5] WORT, C. J. H. and BALMER, R. S. Diamond as an electronic material. Materials Today. [online]. Jan. 2008, vol. 11, no. 1-2, pp. 22-28. Available from: https://doi.org/10.1016/S1369-7021(07)70349-8.

[6] STROBEL, P., RIEDEL, M., RISTEIN, J. and LEY, L. Surface transfer doping of diamond. 2004, vol. 430, p. 3.

[7] FIZZOTTI, F., LO GIUDICE, A., MANFREDOTTI, CH., MANFREDOTTI, C., CASTELLINO, M. and VITTONE, E. Diamond surface conductivity after exposure to molecular hydrogen. Diamond and Related Materials. [online]. Apr. 2007, vol. 16, no. 4-7, pp. 836-839. Available from: https://doi.org/10.1016/j.diamond.2006.11.094.

[8] ČERMÁK, J. et al. Microscopic Electrical Conductivity of Nanodiamonds after Thermal and Plasma Treatments. MRS Advances. [online]. 2016, vol. 1, no. 16, pp. 1105-1111. Available from: https://doi.org/10.1557/adv.2016.112.

[9] MATUNOVÁ, P., JIRÁSEK, V. and REZEK, B. DFT calculations reveal pronounced HOMO-LUMO spatial separation in polypyrrole-nanodiamond systems. Physical Chemistry Chemical Physics. [online]. 2019, vol. 21, no. 21, pp. 11033-11042, Available from: https://doi.org/10.1039/C8CP07622G.

[10] JUNG, H., MIN, H. and GAB, J. Effects of Surface Treatment on Work Function of ITO (Indium Tin Oxide) Films. Journal of the Korean Physical Society. 2005, vol. 47, p. 5.

[11] KIM, J. S. et al. Kelvin probe and ultraviolet photoemission measurements of indium tin oxide work function: a comparison. Synthetic Metals. [online]. Jun. 2000, vol. 111-112, pp. 311-314. Available from: https://doi.org/10.1016/S0379-6779(99)00354-9.

[12] ČERMÁK, J., REZEK, B., KROMKA, A., LEDINSKÝ, M. and KOČKA, J. Electrochemical synthesis and electronic properties of polypyrrole on intrinsic diamond. Diamond and Related Materials. [online]. Sep. 2009, vol. 18, no. 9, pp. 1098-1101. Available from: https://doi.org/10.1016/i.diamond.2009.01.035.

[13] REZEK, B., NEBEL, C. E. and STUTZMANN, M. Hydrogenated diamond surfaces studied by atomic and Kelvin force microscopy. Diamond and Related Materials. [online]. Apr. 2004, vol. 13, no. 4-8, pp. 740-745. Available from: https://doi.org/10.1016/j.diamond.2003.11.051.

[14] CHANG, Y.-C., MILIAIEVA, D. and REZEK, B. Study of photovoltage stability on nanodiamond-polypyrrole composites by Kelvin probe method. In: 10th anniversary international conference on nanomaterials - research \& application (NANOCON 2018). Brno, Czech Republic: TANGER Ltd., 2019, pp. 164-168. 